\title{
Protocol for systematic literature review on implementation of cervical cancer screening and associated factors in Nepal from 2000 to 2018
}

\author{
Aamod D Shrestha ${ }^{1}$, Johanne G Andersen ${ }^{2}$, Dinesh Neupane ${ }^{3}$, Sarita Ghimire ${ }^{4}$, Christine Campbell, Per Kallestrup ${ }^{2}$ \\ ${ }^{1}$ Center for Global Health, Department of Public Health, Aarhus University, Denmark; Nepal Development Society, Bharatpur, Nepal, ${ }^{2}$ Center for \\ Global Health, Department of Public Health, Aarhus University, Denmark, ${ }^{3}$ Nepal Development Society, Bharatpur, Nepal; Johns Hopkins Bloomberg \\ School of Public Health, Baltimore, Maryland, USA, ${ }^{4}$ Nepal Cancer Care Foundation, Lalitpur, Nepal, 5 Usher Institute, University of Edinburgh, \\ Edinburgh, UK \\ Keywords: nepal, cancer prevention, cancer screening, cervical cancer \\ https://doi.org/10.29392/001c.12505
}

\section{Journal of Global Health Reports}

Vol. 4, 2020

\begin{abstract}
Background
Cervical cancer is a major cause of cancer death among women in Nepal. The high burden of this disease necessitates identifying relevant evidence to inform policy development and guidelines. This protocol describes a planned systematic review that will collate and report the experiences of implementation of cervical cancer screening and associated factors in Nepal over the last two decades to identify knowledge gaps to inform future implementation strategies.
\end{abstract}

\begin{abstract}
Methods and analysis
This protocol was developed according to the Preferred Reporting Items for Systematic Review and Meta-Analysis Protocols (PRISMA_P) statement and will apply eligibility criteria to screening and select peer-reviewed research articles and grey literature. A computer-based search will be conducted for each type of publication in the PubMed/ MEDLINE, CINAHL, Scopus and EMBASE databases using various search terms. Search terms will be modified according to each database and the reference lists of the included studies will be screened to identify additional relevant materials. Data synthesis will use narrative synthesis and meta-analysis where appropriate.
\end{abstract}

\section{Ethics and dissemination}

This study does not require ethical approval as only secondary data from published and grey literature will be assessed. The review will be published in a peer-reviewed scientific journal.

PROSPERO registration number: CRD42019144645.

Worldwide, cervical cancer is common and more than $85 \%$ of cases occur in the low- and middle-income countries. ${ }^{1}$ The global estimate from 2018 reported 569,847 new cases and 311,365 deaths due to cervical cancer. ${ }^{1}$ Cervical cancer is the third major cause of cancer death among women in Southeast Asia, and a major cause of cancer death among women in Nepal with an estimated 2,942 new cases and 1,928 deaths in $2018 .{ }^{1}$ Cervical cancer screening is one of the most effective tools for early diagnosis and prevention. 2,3

In Nepal, The Family Health Division developed the first National Guideline for Cervical Cancer Screening and Prevention (CCSP) in 2010. ${ }^{4}$ The annual report from 2016/17 from the Department of Health Services describes the expansion of the cervical cancer screening programme to 64 districts in Nepal. ${ }^{5}$ However, little is known about the experiences related to implementation of cervical cancer screening programmes in diverse settings in Nepal. The high burden of cervical cancer in Nepal, calls for scientific evidence to guide the development of appropriate interventions to increase cervical cancer screening coverage.

Systematic reviews from low- and middle-income countries (LMICs) report that costs, inadequate knowledge, geographical location, cultural views, and lack of understanding of the role of screening are common barriers to effective screening coverage. ${ }^{6,7}$ Family/spouse support, personal or family experiences with cervical cancer or cervical cancer screening, recommendations by healthcare workers, formal employment, and access to cervical cancer screening have been reported as facilitators. ${ }^{8}$ However, little is known about the barriers to and facilitators of cervical cancer screening in Nepal.

There is a need to identify and synthesise country-specific information and describe the status of cancer research to provide evidence that will inform current and future policies, research activities and priorities. This protocol describes a planned systematic review to collate and report experiences related to implementation of cervical cancer screening and associated factors (such as barriers and facilitators) in Nepal. Further, the review will elaborate on the 
understanding of the components involved in conducting a community-based education intervention to increase cervical cancer screening coverage. The findings from the systematic review will provide insights into the status of cervical cancer screening delivery and coverage as well as an understanding of the barriers and facilitators to screening participation across the country. Furthermore, these insights will direct development of future interventions to increase cervical cancer screening coverage. The specific objectives of the planned systematic review are listed below.

\section{OBJECTIVES OF THE PLANNED SYSTEMATIC REVIEW}

- Identify relevant peer-reviewed and grey literature describing cervical cancer screening delivery and coverage among women in Nepal

- Describe and synthesise the experiences of implementing cervical cancer screening in Nepal

- Identify any barriers to and facilitators of cervical cancer screening coverage in Nepal described in the literature

- Collate major findings and recommendations on cervical cancer screening from studies conducted in Nepal from 1 January 2000 to 31 December 2018.

\section{METHODS AND ANALYSIS}

\section{PATIENTS AND PUBLIC INVOLVEMENT}

Patients and the public are not involved in this study.

\section{GUIDELINES AND REGISTRATION}

This protocol was developed in accordance with the Preferred Reporting Items for Systematic Review and MetaAnalysis Protocols (PRISMA-P) statement, and the review that is to be guided by this protocol will be carried out in accordance with PRISMA guidelines. ${ }^{9,10}$

The systematic review has been registered with PROSPERO (CRD42019144645).

\section{ELIGIBILITY CRITERIA}

The following inclusion and exclusion criteria will be used to identify relevant studies:

Population: Studies having a study location or location of sample collection in Nepal, and/or study population of Nepalese women or women living in Nepal.

Intervention: No limitation on the intervention will be applied and non-intervention studies will be eligible.

Context: Studies on cervical cancer screening, barriers, and facilitators associated with cervical cancer screening.

Objective/outcome: Studies with objectives related to and providing information on cervical cancer screening delivery, coverage, barriers, and facilitators associated with cervical cancer screening.

Study design: All designs are eligible.

Types of material:

Published full-text studies in English language between 1 January 2000 and 31 December 2018.

Research reports published by a research team or as a result of research activity.
Electronic copy of theses on cervical cancer screening coverage, barriers and facilitators associated with cervical cancer screening in Nepal on the Tribhuvan, Kathmandu, Pokhara and Purbanchal Universities website.

Government and non-governmental organisational publications and reports directly related to the research publication.

Grey literature that fits the eligibility criteria will be included and presented separately.

Conference presentations or abstracts will not be eligible.

Multi-country studies without separate data from Nepal will not be eligible.

\section{INFORMATION SOURCES}

A computer-based search will be conducted for each type of publication from 1 January 2000 to 31 December 2018 in the following databases PubMed/MEDLINE, CINAHL, Scopus and EMBASE using various search terms. Google search engine, google scholar, government, non-government and universities websites will be searched to find specific grey literature, theses, and research-based reports.

\section{SEARCH STRATEGY}

The search strategies will involve searching within the search fields Titles and Abstracts by two reviewers, using the same appropriate terms/keywords according to the requirements of each databases and websites. The search strategy for PubMed (presented below) will be modified to make it appropriate for search in each database. A filter by year (1 January 2000 to 31 December 2018) will be applied for the search in each database. For each database or website searched, the related search term and the number of studies retrieved and/or screened will be recorded. The search for peer-reviewed literature in PubMed will e.g. be conducted with the following search strategy:

Uterine cervical neoplasms OR Cervical-Cancer OR Cervical-Neoplasm OR Cervical-Neoplasms OR Cancer-ofCervix OR Cervical-cancer-screening OR Visual-Inspection OR Papanicolaou test OR Pap-Smear OR Colposcopy OR Human papillomavirus DNA tests OR HPV-DNA-Test OR Cryotherapy OR Electrocoagulation OR Thermocoagulation OR Conization OR Papillomavirus vaccines OR HPV OR Papillomaviridae OR Human_Papilloma_Virus AND Humans AND Nepal.

A separate search will be performed using the same search strategy as shown above for the PubMed search with Boolean commands; additional modification may be applied to the Google search engine and targeted websites or databases to be searched.

\section{STUDY RECORDS}

\section{DATA MANAGEMENT AND EXTRACTION}

The database search results by two independent researchers will be stored on their computers. Search results of the identified titles and abstracts will be merged and managed in Endnote. Each of the merged studies will be further screened, and full-text documents will be downloaded and 
arranged in a directory according to the type of publication (peer-reviewed article, thesis, grey literature, reports, etc.). Any disagreement between the two independent researchers will be resolved in an open discussion. However, if agreement is not reached, a third independent reviewer will be consulted.

\section{SELECTION PROCESS}

Each search result will be screened following a stepwise process. The titles of the resulting peer-reviewed articles, theses and/or abstracts will be reviewed, and if selected saved in the researcher's account for each database searched. The citation records will then be exported to Endnote. The merged records of the two researchers in Endnote will be assessed and duplicates removed. Abstracts of each study will be screened by applying the eligibility criteria and noted. Studies with full-text documents will be further screened and eligibility criteria will be applied. Each researchers will independently perform these activities. All disagreements (including those related to merging, screening, and selection of search results) will be resolved in an open discussion between the two researchers. Unresolved disagreement will be resolved by consulting a third independent reviewer. Additional papers will be retrieved through hand-searching of citations and reference list tracking. A PRISMA flow chart diagram will be used to summarise the search and selection strategies. ${ }^{10}$

\section{DATA COLLECTION PROCESS}

Data extracted from the full-text documents will include study id, title, journal name, type of publication, year of publication, year the study was conducted, study location, type of study, target population, study setting, ethical considerations, major research area, major findings, and major recommendations. The data extraction form will be included as supplementary material. Authors may be contacted for further clarification or additional information using the email provided in the studies or by a profile search in Google if needed.

\section{MAJOR DATA ITEMS}

Major findings or data elements will include:

1. The major areas researched: Experience on implementation of cervical cancer screening in Nepal.

2. Quantitative data: age of the study participants, cervical cancer screening coverage - pap smear coverage, visual inspection with acetic acid coverage, barriers, and facilitators in cervical cancer screening.

3. Qualitative data: Associated barriers to and facilitators of screening (cultural, financial, religious, personal, family, and social).

\section{EXPECTED OUTCOME}

\section{MAJOR OUTCOMES}

One of the major outcomes of the intended review will be country-specific information on experiences on implementation of cervical cancer screening in Nepal as well as bar- riers and facilitators associated with cervical cancer screening coverage.

\section{QUALITY ANALYSIS / RISK OF BIAS IN INDIVIDUAL STUDIES}

The two researchers will independently assess the quality of each included publication. Standard quality assessment measures will be performed as applicable. The Critical Appraisals Skills Programme (CASP) checklist for quantitative and qualitative studies will be used to assess the clarity in aims and relevance of the study, the study design, sample size, and data collection method. However, bias may arise when collating information from studies difficult to compare with respect to study population, study type, and study design. A meta-analysis may not be performed in case of bias, and differences in the included studies will be reported. Quantitative information will be reported in ranges.

\section{DATA SYNTHESIS AND ANALYSIS}

Data from the research areas will be summarised in tables, which will present the study id, title, journal name, published year, data collection duration, study settings, population types, study design, sampling methods, sample size, cervical cancer screening coverage as well as associated barriers and facilitators. A narrative synthesis will be based on the summarized table. ${ }^{11}$ We will conduct a meta-analysis after gathering sufficient data. For statistical analysis, STATA 15 (Stata Corp, College Station, TX, USA) will be used and a heterogeneity test will be performed using chi-square statistics. A systematic synthesis approach will be conducted to summarize non-quantitative or descriptive and 'thematic synthesis' approach for qualitative information/findings. 11,12

\section{CONFIDENCE IN CUMULATIVE EVIDENCE}

The Grading of Recommendations Assessment, Development, and Evaluation (GRADE) system will be used when applicable to maintain the confidence in cumulative evidence. ${ }^{13}$

\section{DISCUSSION}

This review will describe and synthesize the experiences of implementation of cervical cancer screening a well as the associated barriers to and facilitators of cervical cancer screening coverage among women in Nepal. Furthermore, we will develop a framework to cover the gaps in the current evidence, drawing on the principles from the framework suggested by Robinson et al. and apply this to the findings from our review. ${ }^{14}$ Global, national and regional cervical cancer prevention and control programs are all guided and reviewed mainly by synthesizing information from the findings gathered from all kinds of studies on cervical cancer. $7,12,15,16$ Identification and collation of research will guide the design of interventions to increase cervical cancer screening coverage among women in Nepal where the disease burden of cervical cancer is on the rise. ${ }^{1}$

Strengths and limitations 


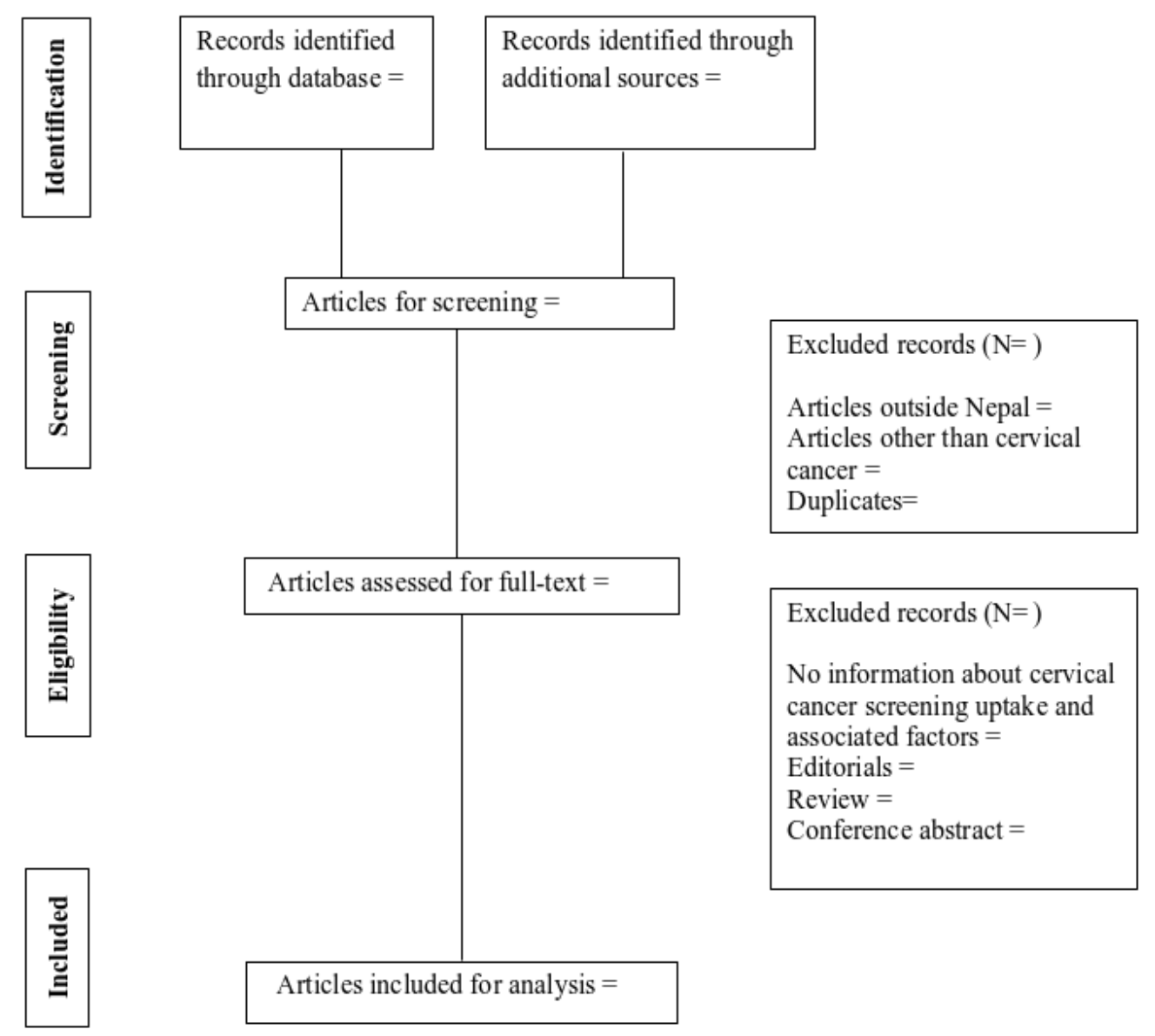

Figure 1. Flow chart.

- The major strength of this protocol is the comprehensive search and screening for eligibility of publications and the inclusion of grey literature, full-text literature and research reports.

- The protocol is designed according to Preferred Reporting Items for Systematic Review and Meta-Analysis Protocols (PRISMA-P) for the development of systematic review protocols.

- The study is limited to published literature in English language.

- Identifying publications from non-indexed databases may be a limitation.

Funding: There is no funding for developing and publication of this protocol paper. However, ADS is a PhD Student at Department of Public Health, Aarhus University, Denmark.

Authorship contributions: ADS made substantial contributions to conception and design of the protocol and wrote the first draft; incorporated coauthors' suggestions; and prepared the final version for submission. CC and PK were involved in the drafting of the protocol, the crosschecking with the PRISMA-P checklist and in revising the protocol critically for important intellectual content. JGA, DN, SG, $\mathrm{CC}$ and PK contributed to all the drafts and approved the final version for submission.

Competing interests: The authors completed the Unified Competing Interest form at www.icmje.org/coi_disclosure.pdf (available upon request from the corresponding author), and declare no conflicts of interest.

\section{Correspondence to:}

Aamod Dhoj Shrestha

Nepal Development Society

Bharatpur-10

Chitwan

Nepal

aamod.shrestha@gmail.com 
This is an open-access article distributed under the terms of the Creative Commons Attribution 4.0 International License (CCBY-4.0). View this license's legal deed at http://creativecommons.org/licenses/by/4.0 and legal code at http://creativecommons.org/licenses/by/4.0/legalcode for more information. 


\section{REFERENCES}

1. Ferlay J, Ervik M, Lam F, et al. Global Cancer Observatory: Cancer Today. Lyon, France: International Agency for Research https://gco.iarc.fr/t oday. Accessed July 24, 2019.

2. Royal Thai College of Obstetricians and Gynaecologists (RTCOG)/JHPIEGO Cervical Cancer Prevention Group: Safety, acceptability, and feasibility of a single-visit approach to cervical cancer prevention in rural Thailand: A demonstration project. Lancet. 2003;361:814-820. doi:10.1016/S014 0-6736(03)12707-9

3. Sankaranarayanan R, Esmy PO, Rajkumar R, et al. Effect of visual screening on cervical cancer incidence and mortality in Tamil Nadu, India: A clusterrandomised trial. Lancet. 2007;370:398-406. doi:10.1 $\underline{016 / S 0140-6736(07) 61195-7}$

4. Government of Nepal, Family Health Division, Ministry of Health and Population. National Guidelines for Cervical Cancer Screening and Prevention in Nepal. Kathmandu; 2010.

5. Government of Nepal, Department of Health Services, Ministry of Health and Population (Nepal). Annual Report of the Department of Health Services of the Fiscal Year 2073/74 (2016/2017). Kathmandu, Nepal; 2017.

6. Islam RM et al. Barriers to Cervical Cancer and Breast Cancer Screening Uptake in Low-Income and Middle-Income Countries: A Systematic Review. Asian Pac J Cancer Prev. 2017;18:1751-1763.

7. Olson B, Gribble B, Dias J, Curryer C, et al. Cervical cancer screening programs and guidelines in low- and middle-income countries. Int J Gynaecol Obstet. 2016;134:239-246. doi:10.1016/j.ijgo.2016.03.011

8. Black E, Hyslop F, Richmond R. Barriers and facilitators to uptake of cervical cancer screening among women in Uganda: A systematic review. BMC Womens Health. 2019;19:108. doi:10.1186/s12905-01 9-0809-z
9. Shamseer L, Moher D, Clarke M, et al. Preferred reporting items for systematic review and metaanalysis protocols (PRISMA-P) 2015: Elaboration and explanation. BMJ. 2015;349(jan02 1):g7647-g7647. do i:10.1136/bmi.g7647

10. Moher D, Liberati A, Tetzlaff J, Altman DG, The PRISMA Group. Preferred Reporting Items for Systematic Reviews and Meta-Analyses: The PRISMA Statement. PLoS Med. 2009;6(7):e1000097. doi:10.137 1/journal.pmed.1000097

11. Schick-Makaroff K, MacDonald M, Plummer M, Burgess J, Neander W. What Synthesis Methodology Should I Use? A Review and Analysis of Approaches to Research Synthesis. AIMS Public Health. 2016;3:172-215. doi:10.3934/publichealth.2016.1.172

12. Thomas J, Harden A. Methods for the thematic synthesis of qualitative research in systematic reviews. BMC Med Res Methodol. 2008;8:45. doi:10.1 186/1471-2288-8-45

13. Mustafa RA, Santesso N, Brozek J, et al. The GRADE approach is reproducible in assessing the quality of evidence of quantitative evidence syntheses. J Clin Epidemiol. 2013;66(736-42):736-742. doi:10.1016/j.jclinepi.201 $\underline{1.06 .009}$

14. Robinson KA, Saldanha IJ, McKoy NA. Development of a framework to identify research gaps from systematic reviews. J Clin Epidemiol. 2011;64:1325-1330. doi:10.1016/j.jclinepi.2011.06.00 9

15. Adams J, Hillier-Brown FC, Moore HJ, et al. Searching and synthesising 'grey literature' and 'grey information' in public health: Critical reflections on three case studies. Syst Rev. 2016;5. doi:10.1186/s136 43-016-0337-y

16. Godin K, Stapleton J, Kirkpatrick SI, et al. Applying systematic review search methods to the grey literature: A case study examining guidelines for school-based breakfast programs in Canada. Syst Rev. 2015;4:138. doi:10.1186/s13643-015-0125-0 